\title{
Effective global conservation strategies
}

\author{
Arising from: R. Grenyer et al. Nature 444, 93-96 (2006).
}

Using data on the global distribution of mammal ${ }^{1}$, bird ${ }^{2}$ and amphibian $^{3}$ species, Grenyer et al. ${ }^{4}$ conclude that planning based on individual taxa does not provide efficient solutions for the conservation of other taxa. They also report that the performance of existing global conservation strategies - endemic bird areas ${ }^{5}$, biodiversity hotspots ${ }^{6}$ and global 200 ecoregions ${ }^{7}$-in representing those taxa is often no better (and in some cases worse) than random. I argue here that the methodology used by Grenyer et al. was not appropriate for purported globally comprehensive analyses. Focusing on analyses of rare species as an example, I demonstrate how the data actually reveal substantial cross-taxon surrogacy and good performance of existing global conservation strategies.

Grenyer et al. describe the scope of their findings as 'global', but their analyses were only performed on subsets of their data. For example, when investigating the congruence between rare birds and rare mammals, they did not obtain a global correlation (across $\sim 19,500$ land-grid cells) but a correlation across cells holding rare species of birds or mammals ( $\sim 5,800$ cells). Hence, they excluded $\sim 13,700$ cells in which there was perfect agreement between rare birds and rare mammals, in that both were absent. Consequently, the correlation coefficient reported $(r=0.48)$ underestimates the true global correlation $(r=0.58)$. The same applies to all other $r$ values reported, including those in their Fig. 2a-c (ref. 4).

The analyses of species numbers expected by chance in areas of variable size (random curves in their Fig. 4; ref. 4) suffer from the same methodological bias: for instance, the curve in their Fig. $4 \mathrm{~b}$ is not for a true random selection, but for a random selection across just the $\sim 6,000$ cells containing rare species. The true global random curve is much shallower (Fig. 1). Globally, endemic bird areas, hotspots and global 200 ecoregions perform substantially better in

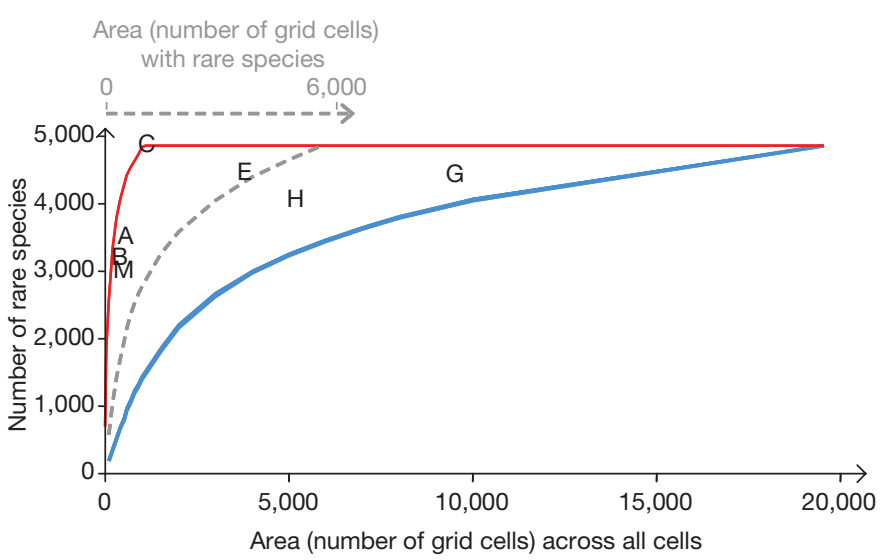

Figure 1 | Relative performance of different types of priority network in capturing rare species (see Fig. 4 b of Grenyer et al. ${ }^{4}$ ). Minimum complementary set representing each species at least once of rare mammals (M), rare birds (B), rare amphibians (A), and rare mammals, rare birds and rare amphibians combined (C). Position is also shown for biodiversity hotspots $(\mathrm{H})$, endemic bird areas (E) and global 200 ecoregions (G). Performance is evaluated by comparing the position of these points for equivalent areas with the maximum number of rare species that can be represented (red line) and the number of rare species expected by chance (blue: $95 \%$ confidence range for randomly selected sets of cells; 100 replicates). The dashed line is the random line from Grenyer et al. ${ }^{4}$, which corresponds to a random selection across just the sites holding rare species, plotted on the dashed grey $x$ axis, top. In Fig. 4 of Grenyer et al. ${ }^{4}$, the position of the random lines therefore cannot be compared with the positions of points $\mathrm{E}, \mathrm{H}$ and $\mathrm{G}$ as they are plotted on different axes (the positions of points $\mathrm{M}, \mathrm{B}, \mathrm{A}$ and $\mathrm{C}$ are the same on both axes). representing rare vertebrate species than would be expected by chance (Fig. 1). Given that hotspots were selected on the basis of plant endemism, this provides evidence for surrogacy of restrictedrange plants in representing rare vertebrates.

Grenyer et al. investigated cross-taxon surrogacy by counting how many target species are represented in minimum complementary sets selected for a particular surrogate taxon (their Table 1; ref. 4). For rare species, they found values ranging from $22.5 \%$ to $77.9 \%$ and concluded that surrogacy is low. However, these values alone are not informative: they need to be compared with what would be expected by chance, and what the maximum possible representation is, in an area of the same size ${ }^{8}$. Figure 1 provides this information for when the target is the representation of rare species across the three groups. It shows that minimum sets representing rare mammals, rare birds or rare amphibians individually represent substantially more overall rare species than would be expected by chance. Furthermore, these minimum sets are noticeably close to the maximum representation possible, which is indicative of a high degree of surrogacy.

Analyses of rare species are the most disrupted by the methodological problems described here, but the other two groups analysed by Grenyer et al. (all species and threatened species) are also affected.

In conclusion, the analyses in Grenyer et al. suffer from a systematic methodological bias that does not allow the results to be compared with the maximal possible representation. The prospects for global conservation planning are, in fact, positive, not dismal as portrayed ${ }^{4}$. It is true that better results will be obtained when highresolution data become available for all taxa we aim to conserve. Nonetheless, at least for the terrestrial realm, good progress can be, and has already been, achieved by conservation planning based on existing data.

\section{METHODS}

The following databases were used: ADHoC database of geographic ranges of birds ${ }^{2}$, owned and developed by the NERC Avian Diversity Hotspots Consortium; global mammal database ${ }^{1,4}$, owned and developed by J. Gittleman; Global Amphibian Assessment ${ }^{3,9}$, developed by SSC-IUCN, CABS-CI and NatureServe. Rare species are those in the lower quartile of the range distribution of each taxonomic group ${ }^{4}$. Optimizations were achieved with the GNU Linear Programming Kit package.

Ana S. L. Rodrigues ${ }^{1}$

${ }^{1}$ Department of Zoology, University of Cambridge, Downing Street, Cambridge CB2 3EJ, UK.

e-mail: aslr2@cam.ac.uk

Received 24 July; accepted 25 September 2007.

1. Sechrest, W. Global Diversity, Endemism and Conservation of Mammals. Thesis, Univ. Virginia (2003).

2. Orme, C. D. L. et al. Global hotspots of species richness are not congruent with endemism or threat. Nature 436, 1016-1019 (2005).

3. Stuart, S. N. et al. Status and trends of amphibian declines and extinctions worldwide. Science 306, 1783-1786 (2004).

4. Grenyer, R. et al. Global distribution and conservation of rare and threatened vertebrates. Nature 444, 93-96 (2006).

5. Stattersfield, A. J., Crosby, M. J., Long, A. J. \& Wege, D. C. Endemic Bird Areas of the World: Priorities for Biodiversity Conservation (BirdLife International, Cambridge, UK, 1998).

6. Mittermeier, R. A., et al. Hotspots Revisited: Earth's Biologically Richest and Most Endangered Terrestrial Ecoregions (CEMEX, Conservation International and Agrupación Sierra Madre, Mexico City, Mexico, 2004)

7. Olson, D. \& Dinerstein, E. The Global 200: Priority ecoregions for global conservation. Ann. Mo. Bot. Gard. 89, 199-224 (2002)

8. Ferrier, S. Mapping spatial pattern in biodiversity for regional conservation planning: where to from here? Syst. Biol. 51, 331-363 (2002).

9. IUCN, Conservation International and NatureServe. Global Amphibian Assessment 〈http://www.globalamphibians.org〉 (2006).

doi:10.1038/nature06374 


\section{Grenyer et al. reply}

\section{Replying to: A. S. L. Rodrigues Nature 450, doi:10.1038/nature06374 (2007).}

Rodrigues $^{1}$ criticizes our demonstration ${ }^{2}$ of low congruence in the global distributions of rare and threatened vertebrates on the grounds that we excluded locations where species counts were zero from our analyses. In practice, this makes no substantive difference to our conclusions. Some sample locations are not inhabited by any organisms of interest: such locations can inflate measures of covariation and association because their values for parameters of interest (in this case, zero counts of species) are identical. This bias has long been known (as 'the double-zero problem's); many ecological techniques and studies exclude double-zero data for this reason.

We are happy to clarify that we excluded double-zero data. We concur with Rodrigues ${ }^{1}$ that including double-zero data increases the observed correlation coefficient $(r)$. Indeed, it cannot fail to do so: $r$ becomes a function of the ratio of double-zero to non-zero data. For example, given our 19,564 terrestrial grid cells, if fewer than 13,043 cells (two-thirds of the world's surface) are filled with perfectly negatively correlated richnesses and the rest with double zeroes, a positive correlation is observed. In any such case, including double-zero data would be to predetermine the result: our comparison of rare mammals and amphibians involved only 3,184 non-zero cells. This is sufficient justification for our choice of method.

Treating all cells as informative, as Rodrigues proposes, becomes analogous to the analysis of binary presence-absence data: it examines associations between cells that have any species of interest, rather than the relative diversities of those cells. Such questions are better addressed by explicitly converting species counts to binary presenceabsence data before analysis. The analyses would rely more on the



Figure 1 | Alternative performance measures. Rodrigues' preferred measure $^{1}$ (blue) evaluates the difference between the maximum possible (upper grey line) and mean random (lower grey line) species capture, given the size of the network in question (black dot). Our preferred measure ${ }^{2}$ (red) evaluates the ratio of species captured to the number of cells in the network. veracity of data on absence, and the metrics of association will depend on the relative abundance of zero to non-zero data.

Rodrigues also criticizes our description of network performance; our two contrasting methods are shown in Fig. 1. Our definition equates performance with the trade-off between network size and species capture. Rodrigues prefers a measure that quantifies the effectiveness of a network given its size. Both measures describe different aspects of reserve network performance. We prefer our definition in this case because we regard the comparison of absolute network sizes to be important. For example, under Rodrigues' definition, the optimal network for rare mammals and the global 200 scheme (points M and G in Rodrigues' Fig. 1; ref. 1) have an effectiveness of around $70 \%$ and $50 \%$, respectively, despite differing in area by more than an order of magnitude.

We agree that good progress has been made in conservation planning and make no claim that the prospects from existing plans are dismal. We do argue, however, that the efficiency of conservation schemes can be improved by detailing where species across multiple higher taxa are found.

Richard Grenyer ${ }^{1}$, C. David L. Orme ${ }^{2}$, T. Jonathan Davies ${ }^{3}$, Sarah F. Jackson ${ }^{4}$, Richard G. Davies ${ }^{5}$, Valerie A. Olson ${ }^{6}$, Kate E. Jones ${ }^{7}$, Kevin J. Gaston ${ }^{4}$, Tim M. Blackburn ${ }^{7}$, Gavin H. Thomas? Peter M. Bennett ${ }^{7} \dagger$, Pamela C. Rasmussen ${ }^{8}$, Tzung-Su Ding ${ }^{9}$, John L. Gittleman ${ }^{10} \&$ Ian P. F. Owens ${ }^{1,2}$

${ }^{1}$ NERC Centre for Population Biology, Division of Biology, Imperial College London, Silwood Park Campus, Ascot, Berkshire SL5 7PY, UK.

e-mail: grenyer@imperial.ac.uk

${ }^{2}$ Division of Biology, Imperial College London, Silwood Park Campus, Ascot, Berkshire SL5 7PY, UK.

${ }^{3}$ National Center for Ecological Analysis and Synthesis, 735 State Street, Suite 300, Santa Barbara, California 93101, USA.

${ }^{4}$ Biodiversity and Macroecology Group, Department of Animal and Plant Sciences, University of Sheffield, Sheffield S10 2TN, UK.

${ }^{5}$ Centre for Ecology, Evolution and Conservation, School of Biological Sciences, University of East Anglia, Norwich NR4 7TJ, UK.

${ }^{6}$ Department of Biology and Biochemistry, University of Bath, Bath BA2 7AY, UK.

${ }^{7}$ Institute of Zoology, Zoological Society of London, Regents Park, London NW1 4RY, UK.

${ }^{8}$ Michigan State University Museum and Department of Zoology, East Lansing, Michigan 48824-1045, USA.

${ }^{9}$ School of Forestry and Resource Conservation, National Taiwan University, Taipei 106, Taiwan.

${ }^{10}$ Odum School of Ecology, University of Georgia, Athens, Georgia 30602-2202, USA.

†Present address: Durrell Institute of Conservation and Ecology, Marlowe Building, University of Kent, Canterbury, Kent CT2 7NR, UK.

1. Rodrigues, A. S. L. Effective global conservation strategies. Nature 450, doi:10.1038/ nature06374 (2007).

2. Grenyer, R. et al. Global distribution and conservation of rare and threatened vertebrates. Nature 444, 93-96 (2006).

3. Legendre, L. \& Legendre, P. Numerical Ecology 2nd edn (Elsevier Science, Amsterdam, 1998).

doi:10.1038/nature06375 